\title{
Growth morphology, structure, and magnetism of ultrathin Co films on W(111)
}

\author{
K. L. Man, ${ }^{1}$ R. Zdyb, ${ }^{2,3}$ S. F. Huang, ${ }^{4}$ T. C. Leung, ${ }^{4}$ C. T. Chan, ${ }^{1}$ E. Bauer, ${ }^{2}$ and M. S. Altman ${ }^{1}$ \\ ${ }^{1}$ Department of Physics, Hong Kong University of Science and Technology, Kowloon, Hong Kong SAR \\ ${ }^{2}$ Department of Physics and Astronomy, Arizona State University, Tempe, Arizona \\ ${ }^{3}$ Institute of Physics, Marie Curie-Skłodowska University, Pl. M. Curie-Skłodowskiej 1, Pl-20031, Lublin, Poland \\ ${ }^{4}$ Department of Physics, National Chung Cheng University, Chia-Yi, Taiwan
}

(Received 8 October 2002; published 2 May 2003)

\begin{abstract}
The growth morphology, structure, and magnetism of ultrathin Co films on the W(111) surface are studied with conventional and spin polarized low energy electron microscopy and diffraction and first principles total energy calculations. Pseudo-layer-by-layer growth of thick pseudomorphic (ps) Co films is observed at $380 \mathrm{~K}$, while Stranski-Krastanov growth and transformation to a $(6 \times 6)$ closed-packed structure are observed at higher temperatures. On the basis of the calculations, only one ps Co monolayer is expected to be thermodynamically stable against three-dimensional island formation. Calculations also indicate that the magnetic moment on Co atoms at the interface is reduced due to strong hybridization with the substrate, but remains non-zero even for the single ps monolayer. Metastable ps $\mathrm{Co} / \mathrm{W}(111)$ is found experimentally to be ferromagnetically ordered at $380 \mathrm{~K}$ when film thickness exceeds 7.6 ps ML. Although only in-plane magnetization is observed, substrate atomic steps have a strong influence on the magnetization easy axis and magnetic domain structure, which suggests that the in-plane magnetocrystalline anisotropy is weak.
\end{abstract}

DOI: 10.1103/PhysRevB.67.184402

PACS number(s): 75.70.Ak, 68.55.Jk, 68.37.Nq, 68.43.Bc

\section{INTRODUCTION}

Sharp W tips have long been used to generate bright and coherent electron beams by vacuum tunneling in field emission for various applications. Tunneling from sharp W tips is also exploited routinely to obtain atomic resolution in scanning tunneling microscopy (STM) and spectroscopy of surfaces. Since W is non-magnetic, electrons that tunnel from this material are unpolarized. It was demonstrated some time ago that spin polarization in field emission could be achieved from $\mathrm{Fe}$ and $\mathrm{Ni}$ single crystal tips ${ }^{1-3}$ and from $\mathrm{W}$ tips coated with thick, bulklike films of $\mathrm{Co}, \mathrm{Ni}, \mathrm{Fe}$, and $\mathrm{Gd}^{3}{ }^{3}$ Some of the recent advances in spin-polarized STM are also based upon $\mathrm{Fe}$ - and Gd-coated W tips. ${ }^{4,5}$ Knowledge of the structural and magnetic properties of magnetic films on $\mathrm{W}$ single crystal surfaces would therefore be useful as a guide for fabricating magnetic films on sharp $\mathrm{W}$ tips and for developing an understanding of spin-polarization effects in tunneling from these structures.

Polycrystalline wires are usually terminated at their tips with a (110) plane. While this may be acceptable for STM, the (111) plane is more appropriate for field emission because of its low work function, ${ }^{6}$ which also happens to be a local minimum. ${ }^{7}$ Furthermore, the (111) emission area will naturally be small on a tip because the surface energy of the (111) plane is higher than those of the other low index planes-(110), (100), and (211) — which dominate the equilibrium crystal shape. ${ }^{8,9}$ A small emission area is desirable from the point of view of source brightness.

Since refractory metals have relatively high surface energies, ${ }^{8}$ it is fairly easy to grow films of lower surface energy materials on top of them in pseudomorphic (ps) or a variety of other ordered structures. Consequently, metal films on low index refractory metal surfaces have been studied quite extensively. ${ }^{10}$ In terms of ferromagnetic materials, the greatest attention has been given to magnetic films on
$\mathrm{W}(110)$ and $\mathrm{W}(100)$ in the past, ${ }^{11-13}$ while significantly less is known about ferromagnets on $\mathrm{W}(111) .{ }^{14-22} \mathrm{~A}$ major focus in the study of metals on the $\mathrm{W}(111)$ surface in recent years has been their capacity to induce microscopic surface faceting. ${ }^{14-19,23}$ Although ordered metal overlayers form at low temperature, the (111) surface is quite prone to facetting in the presence of numerous metal species at high temperature. Facetting occurs predominantly to $\{211\}$ pyramidal structures, although $\{110\}$ faceted structures have also been observed to a lesser extent. Both types of facets have been accounted for by theory. ${ }^{23-25}$ The ferromagnets $\mathrm{Fe}, \mathrm{Co}$, Ni, and $\mathrm{Gd}$ have been found not to induce such facetting. Surprisingly, relatively little work has been carried out on the ferromagnets on $\mathrm{W}(111)$ beyond the issue of facetting, and the little that is known concerning structure does not touch at all on magnetism.

The structure and morphology of Co films on W(111) were reported on briefly by Guan et al. ${ }^{16}$ The key conclusions were that thick, ps films could be grown at room temperature. Material in excess of a stable wetting layer agglomerated into three-dimensional clusters upon annealing above about $500 \mathrm{~K}$, with the most significant morphological change occurring between 500 and $700 \mathrm{~K}$. Low energy electron diffraction (LEED) measurements provided no evidence of facetting induced by Co layers up to what was believed to be four physical monolayers ( $\mathrm{PML}=3 \mathrm{ps} \mathrm{ML}$, at which all exposed substrate atoms in the topmost three "geometric layers" of the (111) surface are covered by adsorbate atoms) and annealing temperatures up to $1200 \mathrm{~K}$.

The aim of the present work is to broaden our knowledge of structure and morphology and to explore several aspects of magnetism of Co films on W(111). Our results are in agreement with the earlier limited work, except with regards to the thickness of the thermodynamically stable wetting layer. We have also observed a massive restructuring of Co films from ps to closed-packed (cp) arrangement above 700 
$\mathrm{K}$, which was not reported before. The magnetic measurements presented here are to our knowledge the first performed on ultrathin films on the W(111) surface. Questions pertaining to the thickness at which Co films become magnetic at room temperature, magnetization easy axis and the influence of surface steps on magnetism are addressed in this work. Answers to these question provide crucial guidance to ongoing work on Co-coated W(111) tips for vacuum tunneling applications.

\section{EXPERIMENTAL STUDIES}

\section{A. Experimental details}

The experiments were carried out in two separate instruments. The work on structure and morphology described in Sec. II B was performed in a conventional low energy electron microscope (LEEM), and the magnetic measurements of Sec. II C were performed in a spin polarized LEEM (SPLEEM). The contrast, resolution and operation principle of LEEM and SPLEEM have been described previously. ${ }^{26-29}$ The conventional LEEM diffraction and phase contrast mechanisms are augmented by magnetic contrast when a spin-polarized electron beam is used to illuminate the surface. Magnetic sensitivity of SPLEEM is based upon spindependent exchange scattering. The exchange asymmetry is defined as

$$
A_{\mathrm{ex}}=\frac{1}{P} \frac{I_{\uparrow}-I_{\downarrow}}{I_{\uparrow}+I_{\downarrow}},
$$

where $I_{\uparrow}$ and $I_{\downarrow}$ represent the reflected intensities for oppositely polarized incident beams, called spin-up and spindown, and $P$ is the degree of polarization of the incident beam. Subtraction of spin-up and spin-down images in the numerator eliminates non-magnetic diffraction and topographical image features that are found in conventional LEEM images and leaves features that originate exclusively in the magnetism of the sample. Division by the spin-up and spin-down image sum yields an asymmetry image in which intensities are proportional to the incident beam spin polarization and the component of the local magnetization vector that lies along the incident beam polarization direction. The SPLEEM that was used in the present investigations was equipped with a spin manipulator, ${ }^{30}$ which can be used to adjust the azimuthal and polar angles of the incident beam spin direction fully relative to its propagation direction. This allows complete characterization of the sample magnetization direction in the in- and out-of-plane directions and tilted directions in between. It is also worth noting that the sum of the spin-up and spin-down images that is found in the denominator of the exchange asymmetry [Eq. (1)] contains only diffraction information and no magnetic information. Variations of diffraction intensity in the denominator occur during film growth that are related to film thickness, for example, periodic intensity oscillations that accompany periodic nucleation, growth and completion of layers. By monitoring diffraction intensity and the evolution of the exchange asymmetry simultaneously and in the identical sample area, film thickness and magnetism can be correlated continuously and with great precision with SPLEEM during growth. ${ }^{31}$

Two samples were used, one of which was well-oriented to within $0.05^{\circ}$ of the [111] direction and nominally flat and the other miscut by less than $1^{\circ}$ with higher step density. On the basis of LEED spot streaking, step edges were determined to be along a direction that is rotated by a little less than $10^{\circ}$ from the in-plane [1 $\left.1 \overline{1} 0\right]$ direction on the vicinal surface. The samples were cleaned by annealing at $1200 \mathrm{~K}$ in an oxygen pressure of $1 \times 10^{-7}$ torr and flashing to $2000 \mathrm{~K}$. In the LEEM work, Co was deposited from an electron beam heated rod in a deposition source that was equipped with flux monitor by proportional ion current measurement. Co was deposited from a directly heated crucible in the SPLEEM work. The base pressure in the LEEM chamber was 1 $\times 10^{-10}$ torr, and in the SPLEEM it was $8 \times 10^{-11}$ torr. The pressure rose to the mid $10^{-10}$-torr range during deposition in both instruments. The sample temperature was measured by a W/Re3\%-W/Re25\% thermocouple attached to the sample holder immediately adjacent to the side of the sample.

\section{B. Structure and morphology}

The structure and morphology of Co films on W(111) were studied in-situ by LEED and LEEM. Two characteristic LEED patterns were observed under different growth conditions - a $(1 \times 1)$ pattern indicative of a pseudomorphic $(\mathrm{ps})$ bcc film, and a $(6 \times 6)$ pattern (Fig. 1). The $(6 \times 6)$ pattern is characterized primarily by sharp satellite spots surrounding integer spots with three-fold rotational symmetry. The separation between satellite and integer spots indicates a sixfold periodicity in the in-plane $\langle 11 \overline{2}\rangle$ substrate directions. Weaker sixfold periodic spots displaced further from the integer spots were also observed. As will be made clear below, the $6 \times 6$ pattern is consistent with a cp hcp(0001) or fcc(111) Co structure.

Integrated intensities of the LEED spots at the (00) and $(5 / 60)$ satellite positions were measured continuously during deposition at temperatures in the range 380-910 K (Fig. 2). The measurements were carried out at $E=8 \mathrm{eV}$. At this energy, electrons penetrate fairly deep below the surface and as a result carry information about the film structure throughout its entire thickness in the thickness range studied. This is supported by observations of quantum size effects in electron reflectivity that were reported for Co on the $\mathrm{W}(110)$ surface. ${ }^{32}$ Therefore, LEED spot intensities vs time, $I(t)$, at very low energy give a good qualitative view of the evolution of the film structure and morphology, although the interpretation of these data is not trivial.

At all deposition temperatures, the (00) beam intensity drops initially during Co deposition and then rises again to an intense peak close to a time that we define as $t_{1}$, after which it drops again to a minimum near zero at a time $t_{2}$ $=2 t_{1}$. At $380 \mathrm{~K}$, the intensity minimum at $t_{2}$ is followed by a linear increase to a sharp break at a time $t_{3}=3 t_{1}$, followed by another linear increase with smaller slope that continues for the remainder of the deposition. The scenario up to $t_{2}$ is followed with minor differences at all deposition tempera- 

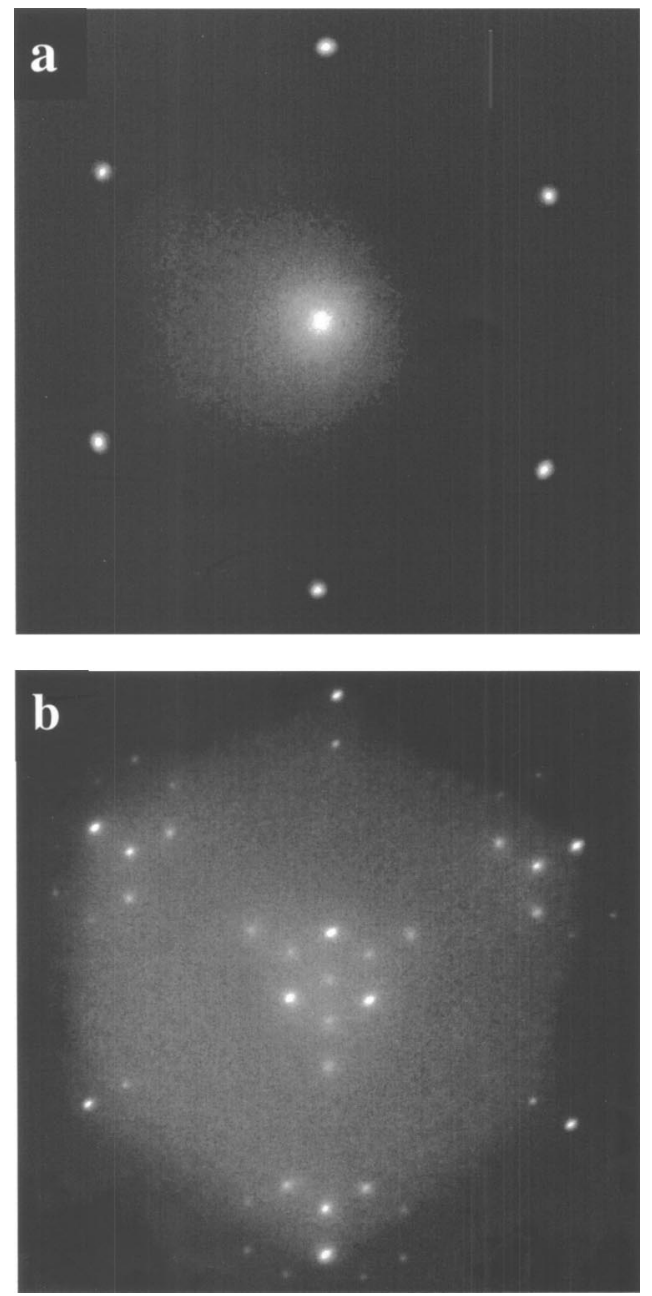

FIG. 1. LEED patterns for Co films on the W(111) surface (a) $(1 \times 1)$ at $12.5 \mathrm{eV}$ and $(\mathrm{b})(6 \times 6)$ at $17.4 \mathrm{eV}$.

tures up to the highest studied, $910 \mathrm{~K}$. Among the differences is a change in the shape of the intensity minimum between the beginning of deposition, $t_{0}=0$, and $t_{1}$. It is worth noting that the intensity variation between $t_{0}$ and $t_{1}$ shows three distinct segments at $T \geqslant 685 \mathrm{~K}$. The height of the intensity peak at $t_{1}$ also decreases as the deposition temperature is increased above $380 \mathrm{~K}$, reaches a minimum value when deposition is carried out at $710-735 \mathrm{~K}$ and then recovers to higher values at higher deposition temperatures [Fig. 3(a)]. A subtle change of shape of the $(00) I(t)$ curve between $t_{1}$ and $t_{2}$ occurs at about $710-760 \mathrm{~K}$, and at $735 \mathrm{~K}$ and above the (00) beam intensity reaches a minimum no longer at $t_{2}$, but at a time that is $7 \%$ longer. Most significantly, the strong recovery of the intensity after $t_{2}$ which is seen at $380 \mathrm{~K}$ becomes less pronounced at higher deposition temperature until essentially no intensity remains in the (00) beam after $t_{2}$ for $T \geqslant 685 \mathrm{~K}$.

Intensity at the $(5 / 60)$ diffraction position is not observed until after the deposition time $t_{1}$ at all deposition temperatures studied. The $(5 / 60)$ intensity then rises to a peak at a time $t_{2}^{*}$ near $t_{2}$. This peak is weak and occurs shortly before $t_{2}$ at $380 \mathrm{~K}$, but is increasingly stronger and shifts to gradually later time at higher deposition temperatures (Figs. 2 and
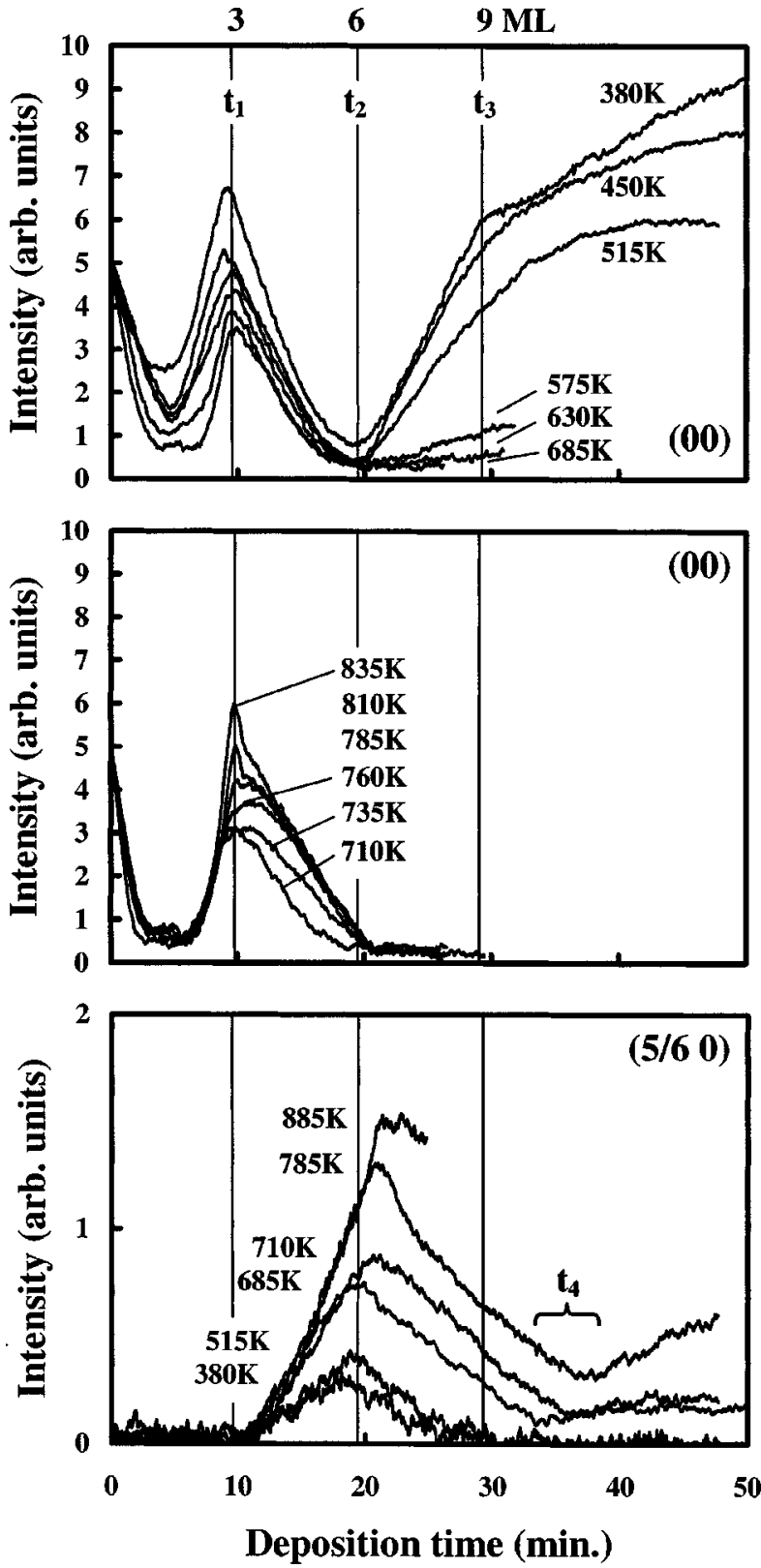

FIG. 2. (00) and (5/6 0) LEED spot integrated intensities vs time during Co deposition at different temperatures. Placement of the temperature labels in the (00) and (5/6 0) plots corresponds to the heights of the peaks at $t_{1}(3 \mathrm{ML})$ and near $t_{2}(6 \mathrm{ML})$, respectively.

$3)$. When the $(6 \times 6)$ structure is better formed above $T$ $\sim 710 \mathrm{~K}$, the $(5 / 60)$ diffraction appears initially at $1.15 t_{1}$ and reaches a peak at $t_{2}^{*}=1.08 t_{2}$ [Fig. 3(b)], which coincides nearly exactly with the disappearance of intensity in the $(00)$ beam above $735 \mathrm{~K}$. Complementary LEEM observations in this temperature range, $T>710 \mathrm{~K}$, reveal that the $(6 \times 6) \mathrm{cp}$ phase grows continuously from small nuclei to cover the surface in the same time frame, $1.15 t_{1}<t<t_{2}^{*}$ (Fig. $4)$. After reaching a peak at $t_{2}^{*}$, the $(5 / 60)$ diffraction intensity decreases again to zero at lower temperatures ( $T$ $\leqslant 655 \mathrm{~K}$ ), where it remains during additional deposition. At intermediate temperatures $(685 \mathrm{~K} \leqslant T \leqslant 810 \mathrm{~K})$, the $(5 / 60)$ intensity passes through a broad minimum at roughly $t_{4}$ 

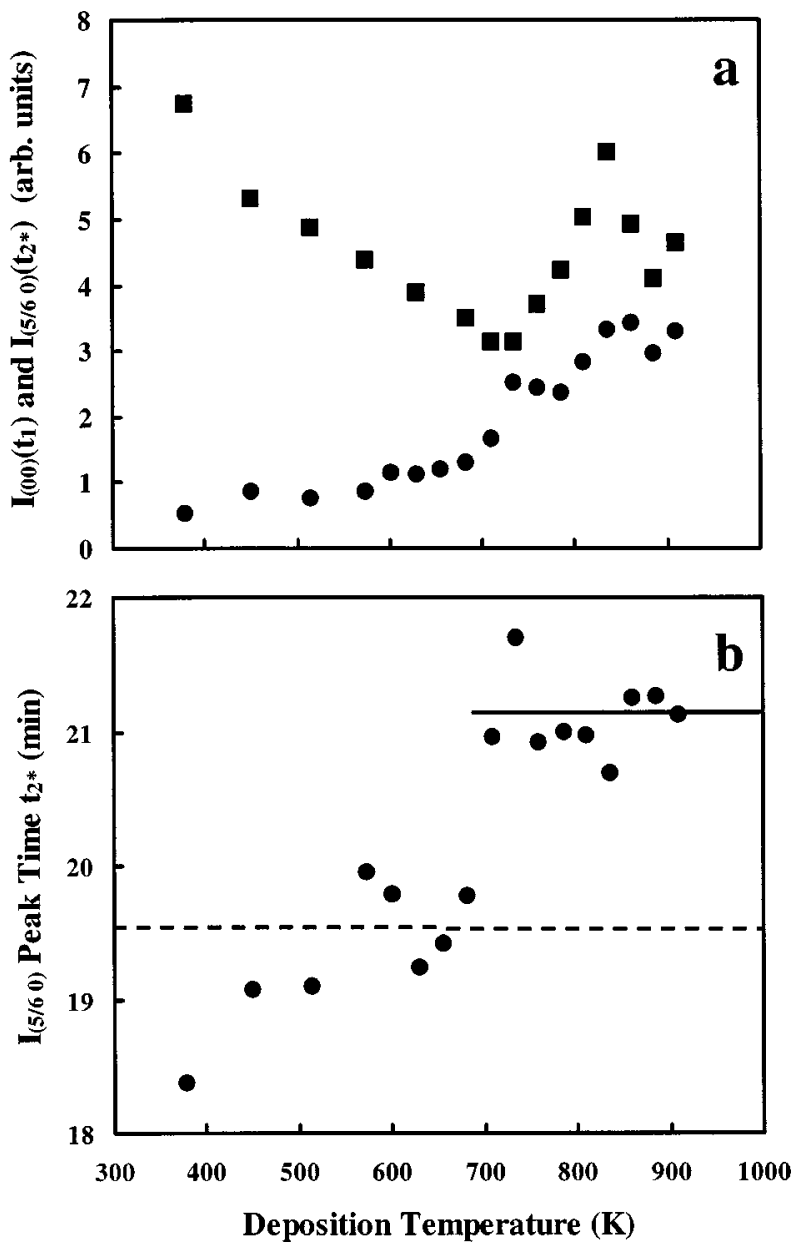

FIG. 3. (a) Integrated intensities of the (00) and (5/6 0) LEED spots at their respective peak times, $I_{(00)}\left(t_{1}\right)(\mathbf{\square})$ and $I_{(5 / 60)}\left(t_{2}^{*}\right)$ $(\mathbf{O})$, and (b) (5/6 0) LEED spot peak time, $t_{2}^{*}$, vs deposition temperature. The horizontal solid line in (b) is the average time $\left\langle t_{2}^{*}\right\rangle$ for deposition at $T \geqslant 710 \mathrm{~K}$. The time $t_{2}$ that corresponds to $6 \mathrm{ps} \mathrm{ML}$ is also indicated by the horizontal dashed line in (b). In (a), $I_{(5 / 60)}$ $\times\left(t_{2}^{*}\right)$ is scaled by $2 x$ compared to Fig. 2 .

$=(3.5-3.9) t_{1}$ before rising again slowly at higher coverages. At higher temperatures $(T \geqslant 840 \mathrm{~K})$, the $(5 / 60)$ diffraction intensity does not decrease as significantly after its peak at $t_{2}^{*}$ as it does at intermediate temperature.

\section{Magnetism}

SPLEEM investigations were concentrated on magnetic properties of ps films, with the goal of growing continuous ps films that were thick enough to have a Curie temperature exceeding room temperature. At temperatures where the cp structure is best formed, $T>710 \mathrm{~K}$, there is an additional risk of three-dimensional bulk alloying that is expected to disrupt film magnetism. Most of the results presented here were obtained on the vicinal surface with high step density. Refer to the discussion in Sec. IV A for an explanation of the coverage assignments.

The influence of growth conditions upon magnetism was investigated by preparing ps films in different ways: (1) deposition entirely at $380 \mathrm{~K}$, and (2) deposition of the first 3

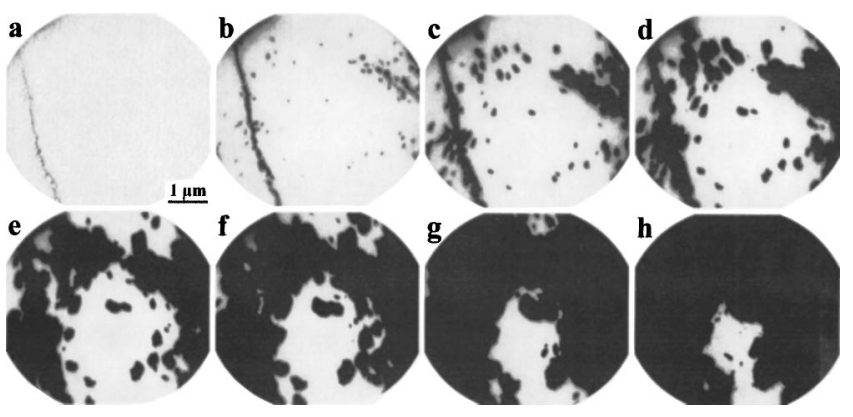

FIG. 4. LEEM images of the $\mathrm{W}(111)$-Co $(1 \times 1)$ to $(6 \times 6)$ transformation taken continuously during deposition at $T=810 \mathrm{~K}$. The Co coverages in images (a)-(h) are equivalent to $3.3,3.5,4.0,4.5$, $5.0,5.5,6.0$, and $6.3 \mathrm{ps}$ ML, respectively [imaging energy $=20.0 \mathrm{eV} ;(1 \times 1)$, white; $(6 \times 6)$, black].

ps $\mathrm{ML}$ at 685 or $810 \mathrm{~K}$ with subsequent deposition carried out at $380 \mathrm{~K}$. These two procedures were each carried out at two deposition rates, 0.23 and $0.46 \mathrm{ML} / \mathrm{min}$. The aim of depositing initially at elevated temperature in procedure (2) was to improve ps ordering during the formation of the interface. Growth of ps films beyond 3 ps ML at elevated temperature was not possible because of nucleation of the $(6 \times 6)$ cp phase.

Exchange asymmetry [Eq. (1)] and diffraction intensity oscillations were continuously and simultaneously monitored on precisely the same area during deposition, as outlined in Sec. II A. An example of the correlated measurement of magnetism and thickness on the vicinal surface is shown in Fig. 5. For this measurement, the first 3 ps ML were deposited at $810 \mathrm{~K}$ and subsequent ps layers were deposited at $380 \mathrm{~K}$ at a rate of $0.23 \mathrm{ML} / \mathrm{min}$. The time indicated on the $x$-axis represents the total deposition time for high and low temperature stages. The first magnetic signal that is reliably above the noise level in Fig. 5(b) occurs at 32.2 min or 7.40 ps ML. Similar asymmetry curves were obtained for films prepared under all conditions studied. From several experiments on films prepared in different ways, the onset of magnetism at $380 \mathrm{~K}$ is determined to be at $7.60 \pm 0.15 \mathrm{ps}$ ML. The magnitude of the exchange asymmetry was also found to be similar for all growth conditions, but with a tendency for larger asymmetry when the first 3 ps ML were deposited at $810 \mathrm{~K}$, irrespective of the deposition rate. The use of a higher deposition rate was also found to yield more reproducible exchange asymmetry results. However, these results must be interpreted cautiously, because the exchange asymmetry measurement depends not only upon the sample magnetization, but also upon the incident beam spin polarization. Control measurements revealed that the reproducibility of the incident spin polarization was a little better than the variation of the exchange asymmetry caused by the different deposition conditions.

Only in-plane magnetization was found for all preparation conditions on both the well-oriented and the vicinal W(111) surfaces. In view of the threefold symmetry of the W(111) surface, a threefold anisotropy would be expected, while only a uniaxial anisotropy was observed on the vicinal surface with one of the three in-plane $\langle 11 \overline{2}\rangle$ directions as easy 

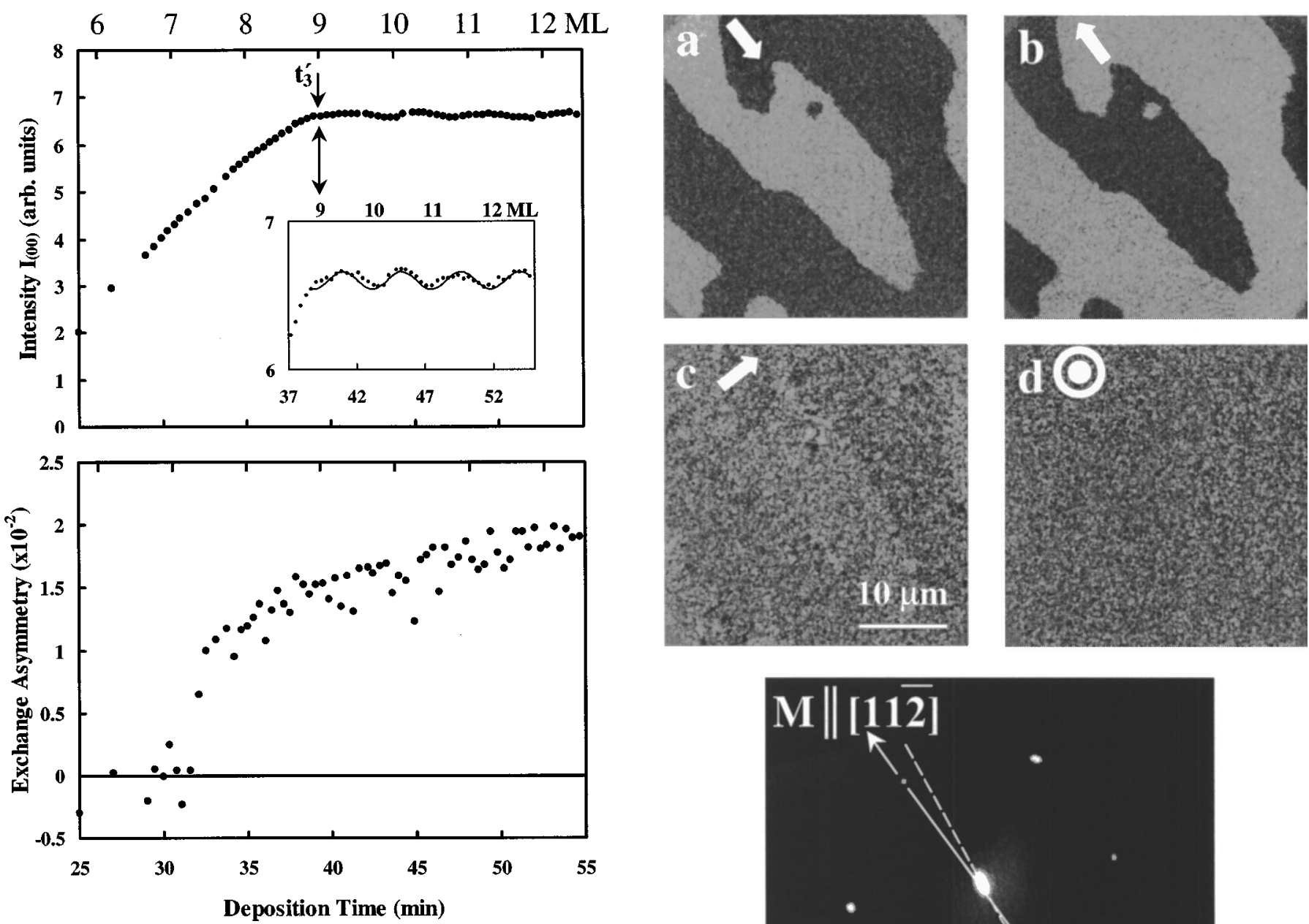

FIG. 5. Variations of (a) SPLEEM sum image intensity $\left\langle I_{\uparrow}\right.$ $\left.+I_{\downarrow}\right\rangle$ and (b) exchange asymmetry [Eq. (1)] with time/thickness during deposition at $T=380 \mathrm{~K}$. The inset in (a) shows weak intensity oscillations due to layer-by-layer growth. A sinusoidal function is fit to the oscillations to determine the deposition rate. The inflection at $t_{3}^{\prime}(9 \mathrm{ML})$ corresponds to a similar feature at $t_{3}$ of the $(00)$ intensity in Fig. 2.

axis (Fig. 6). The $(\varphi, \theta)$ notation in the figure caption refers to the polarization direction of the incident beam, and therefore the sample magnetization component that is being probed. The polar angle $\theta$ is referenced to the surface normal, $\theta=0^{\circ}$ being out of plane and $90^{\circ}$ in plane. Variation of the azimuthal angle $\varphi$ probes different in-plane directions. The arrows in each image indicate the incident beam polarization directions that correspond to the $(\varphi, \theta)$ settings. Magnetic contrast is strongest in the directions labeled $(\varphi, \theta)$ $=\left(120^{\circ}, 90^{\circ}\right)$ and $\left(300^{\circ}, 90^{\circ}\right)$ and is absent at the orthogonal in-plane $\left(210^{\circ}, 90^{\circ}\right)$ and out-of-plane $\left(120^{\circ}, 0^{\circ}\right)$ directions. The possibility that the uniaxial magnetization may be caused by a step-induced anisotropy is indicated by the streaking of the (00) LEED spot nearly parallel to the magnetization direction (Fig. 6). Surface steps also had a significant impact upon the magnetic domain structure. A clear tendency for elongated domains sometimes reaching millimeter in size and with domain walls preferentially parallel to the magnetization direction were evident.

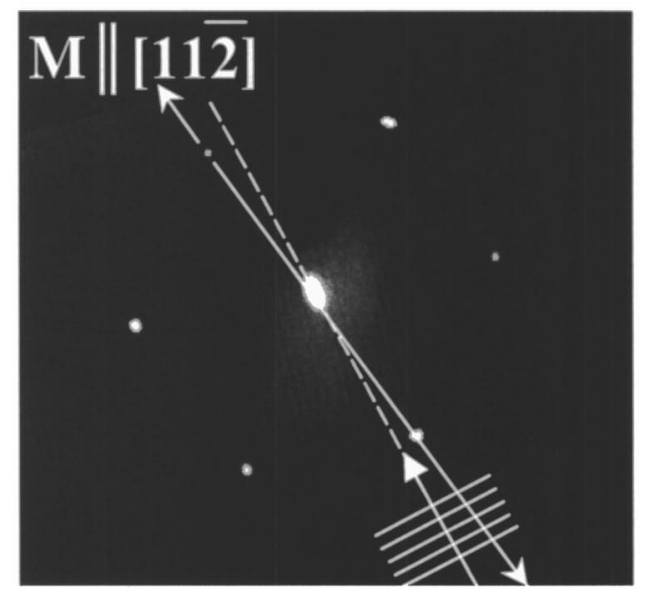

FIG. 6. SPLEEM images of in-plane magnetic domains in ps Co films on vicinal $\mathrm{W}(111)$ as a function of incident beam polarization direction (arrows) specified by $(\varphi, \theta)$ in (a) $\left(120^{\circ}, 90^{\circ}\right)$, (b) $\left(300^{\circ}\right.$, $\left.90^{\circ}\right)$, (c) $\left(210^{\circ}, 90^{\circ}\right)$, and (d) $\left(120^{\circ}, 0^{\circ}\right)$ [imaging energy $4.5 \mathrm{eV}$ ]. The LEED pattern of vicinal W(111) is shown below with magnetization $(M)$ direction and in-plane direction perpendicular to steps specified by the solid and dashed lines, respectively.

In contrast, a more complex multi-domain structure with considerably smaller features, whose size and shape also depended strongly on the surface condition of the substrate, was observed on the well-oriented W(111) surface (Fig. 7). Except at defects, the domain boundaries were never sharp. In addition, significant deviation from the expected threefold symmetry was observed on the well-oriented surface. Magnetization was found to lie along both the $\langle 11 \overline{2}\rangle$ and $\langle 1 \overline{1} 0\rangle$ in-plane directions (see Fig. 7). The dependence of the magnetization upon film thickness, however, was similar to the behavior on the vicinal surface and thus was not influenced by the details of the domain structure. 

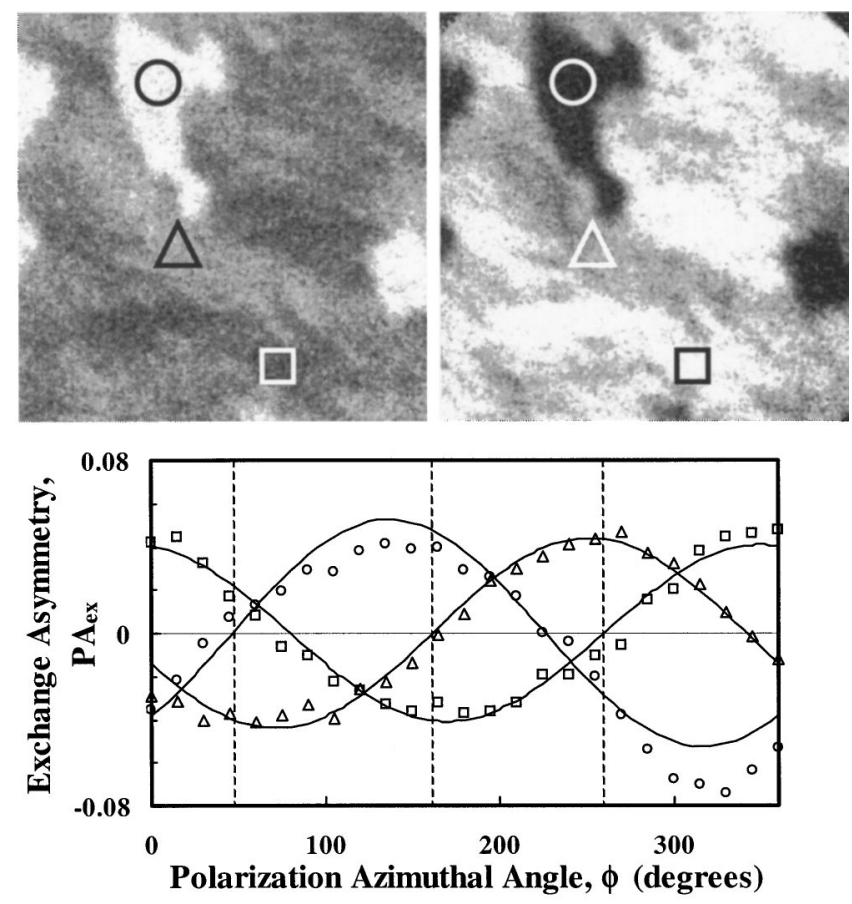

FIG. 7. SPLEEM images of in-plane magnetic domains on a well-oriented W(111) surface for incident polarization oriented inplane with $(\varphi, \theta)$ specification (left) $\left(180^{\circ}, 90^{\circ}\right)$ and (right) $\left(0^{\circ}\right.$, $90^{\circ}$ ). The dependence of the exchange asymmetry [Eq. (1)], $P A_{\mathrm{ex}}$, upon polarization azimuthal angle, $\varphi$, for the three domains identified in the images by the symbols is shown below with the corresponding symbols. The vertical dashed lines identify the corresponding $A_{e x}=0$ conditions at $\phi=47^{\circ}(\bigcirc), 162^{\circ}(\triangle)$ and $260^{\circ}(\square)$ for each domain. The interval between $\square$ and $\bigcirc, \triangle$ deviates from three-fold symmetry by $30^{\circ}$ [imaging energy $3.6 \mathrm{eV}$ ].

\section{THEORETICAL STUDIES}

Theoretical calculations were performed for ps Co films on $\mathrm{W}(111)$ and $\mathrm{W}(110)$ for comparison. The calculations were done using the full potential linear augmented plane wave method ${ }^{33}$ employing the Perdew, Burke, Ernzerhof form of the generalized gradient approximation. ${ }^{34}$ The calculated moment of bulk hcp Co is $1.70 \mu_{B}$, which is in good agreement with the experimental value of $1.714 \mu_{B} \cdot{ }^{35}$ The surfaces were simulated by the standard repeated slab geometry. Different slab thicknesses were used for the different orientations. We increased the slab thickness until convergence was found. We found that seven layer slabs and eleven layer slabs are needed for converged results for the (110) and (111) orientations, respectively. The Co overlayers were added pseudomorphically on both sides of the slab. The vacuum thickness was about 18 a.u. We restrict calculations to $(1 \times 1)$ unit cells. We found that the final values of the magnetic moment are rather sensitive to the atomic positions. All the interlayer distances were fully relaxed by computing the Hellmann-Feynmann forces, until the maximum force acting on the atoms was smaller than $5 \mathrm{mR} / \mathrm{a}$.u. Different starting atomic geometries and initial values of the magnetic moments were used to test the results for local vs. global energy minimum character and to ensure that we land consistently on the same (true) ground state after iteration to self-consistency. The radii of the muffin-tin spheres were set to 2.3 a.u. for $\mathrm{W}$ and 2.0 a.u. for Co. For $k$-point sampling, we used a uniform $16 \times 16 \times 1$ grid for $\mathrm{W}(110)$ and a 12 $\times 12 \times 1$ grid for $\mathrm{W}(111)$ in the Brillouin zones.

The results for magnetic moments, heats of formation $(H)$ and relaxation geometries for $\mathrm{Co} / \mathrm{W}(111)$ and $\mathrm{Co} / \mathrm{W}(110)$ are shown in Tables I and II, respectively. The heats of formation, $H$, given in $\mathrm{eV}$ per atom, are stated relative to the bulk cohesive energies of $\mathrm{Co}$ and the heat of formation of the slab. Therefore, negative heats of formation for a given thickness in Tables I and II indicate that a complete, wetting layer with that thickness is thermodynamically stable. Positive heats of formation indicate that layers in excess of the thickest thermodynamically stable wetting layer are unstable with respect to three-dimensional island formation. The leftmost and rightmost Co moments that are indicated in the table correspond to the surface layer and the interface layer, respectively. The relaxations of interlayer spacings are stated in Tables I and II with respect to the bulk W(111) and W(110) layer spacings, respectively, where $\Delta d_{0}$ is the relaxation of the interface layer spacing between Co and $\mathrm{W}$ and $\Delta d_{n}$ and $\Delta d_{-n}$ are for the layer spacings removed by $n$ layers from the interface in the Co film and W substrate, respectively. The (111) surface has substantially stronger relaxations due to its more open atomic structure. Strong relaxation of the (111) orientation has also been noted in previous local density functional and empirical potential calculations for clean $\mathrm{W}(111)^{36,37}$

\section{DISCUSSION}

\section{A. Structure and morphology}

Pseudomorphic and $(6 \times 6)$ periodic film structures have been identified here experimentally. Pseudomorphic films were reported previously, ${ }^{16}$ while the $(6 \times 6)$ structure was not identified prior to our work. Instead, a $(1 \times 1)$ diffraction pattern was presented in Ref. 16 for a Co film that had been annealed to $1100 \mathrm{~K}$ as representative evidence of pseudomorphic films formed under all conditions studied.

TABLE I. Magnetic moments, heats of formation $(H)$ and relaxations relative to bulk W layer spacing for $\mathrm{ps} \mathrm{Co} / \mathrm{W}(111)$.

\begin{tabular}{|c|c|c|c|c|c|c|c|c|c|c|c|}
\hline \multirow[b]{2}{*}{$\mathrm{W}(111)$} & \multicolumn{4}{|c|}{ Magnetic moment $\left(\mu_{B}\right)$} & \multirow{2}{*}{$\begin{array}{c}H \\
(\mathrm{eV})\end{array}$} & \multicolumn{6}{|c|}{ Relaxation (\%) } \\
\hline & & Co & & W & & $\Delta d_{2}$ & $\Delta d_{1}$ & $\Delta d_{0}$ & $\Delta d_{-1}$ & $\Delta d_{-2}$ & $\Delta d_{-3}$ \\
\hline Co & & & 0.74 & -0.03 & -0.58 & & & -43.7 & -12.6 & 2.6 & 8.0 \\
\hline $2 \mathrm{Co}$ & & 1.64 & 0.87 & -0.01 & 0.01 & & -9.1 & -55.5 & 19.5 & -3.1 & 1.9 \\
\hline $3 \mathrm{Co}$ & 1.66 & 1.86 & 1.49 & -0.01 & 0.57 & -29.5 & -12.4 & -14.7 & -7.4 & -0.2 & 0.1 \\
\hline
\end{tabular}


TABLE II. Magnetic moments, heats of formation $(H)$, and relaxations relative to bulk W layer spacing for $\mathrm{ps} \mathrm{Co} / \mathrm{W}(110)$.

\begin{tabular}{|c|c|c|c|c|c|c|c|c|}
\hline \multirow[b]{2}{*}{$\mathrm{W}(110)$} & \multicolumn{3}{|c|}{ Magnetic moment $\left(\mu_{B}\right)$} & \multirow{2}{*}{$\begin{array}{c}H \\
(\mathrm{eV})\end{array}$} & \multicolumn{4}{|c|}{ Relaxation (\%) } \\
\hline & & & $\mathrm{W}$ & & $\Delta d_{1}$ & $\Delta d_{0}$ & $\Delta d_{-1}$ & $\Delta d_{-2}$ \\
\hline Co & & 1.40 & -0.03 & -0.10 & & -15.2 & -2.2 & 1.9 \\
\hline $2 \mathrm{Co}$ & 2.00 & 1.69 & 0.02 & 0.28 & -25.2 & -8.0 & 2.0 & 2.8 \\
\hline
\end{tabular}

However, faint satellite diffraction spots adjacent to integer spots are evident in the published LEED pattern ${ }^{16}$ but were not pointed out by the authors. ${ }^{38}$ These satellite spots occur at positions that correspond to the strong $(6 \times 6)$ diffraction observed here.

A $(6 \times 6)$ periodic Co film can be formed by lateral compression of a bulk hcp or fcc closed packed plane by $2.8 \%$. This reduces the in-plane lattice constant from the bulk value of $a=2.507$ to $2.438 \AA$, and gives rise to coincidence between 11 Co spacings and six W spacings. Lateral contraction of a two-dimensional film from the bulk configuration is generally expected. In a macroscopic picture, we can attribute such a contraction to the surface stress. At the microscopic level, we may argue using effective-medium type theory that there is a specific preferred electronic charge density for a certain element at its atomic site when the system is at equilibrium, and a reduction in coordination number (due to the presence of a surface) would bring the electronic density to a smaller value, which could be compensated for by a contraction.

An alternative explanation of the $(6 \times 6)$ diffraction pattern is that it arises from alloying between $\mathrm{Co}$ and $\mathrm{W}$. This explanation can be excluded because the same or similar cp diffraction pattern was observed in the low temperature range for $\mathrm{Fe}$ and $\mathrm{Ni}$ on W(111) while evidence for two-dimensional (2D) alloy formation was found in these systems only upon annealing to $750-800 \mathrm{~K} .{ }^{14}$ Formation of 3D alloy clusters can also be excluded as an explanation of the $(6 \times 6)$ phase because of LEEM observations of the rapid lateral growth of this phase over the whole surface during the deposition of the equivalent of $1 \mathrm{cp} \mathrm{ML} \mathrm{of} \mathrm{Co} \mathrm{(see} \mathrm{Fig.} \mathrm{4).} \mathrm{Nucleation} \mathrm{and}$ slow lateral growth of 3D alloy clusters were indeed observed, but only after deposition of the equivalent of roughly $5 \mathrm{cp} \mathrm{ML}$ at $810 \mathrm{~K}$. That is well after the $(6 \times 6)$ structure completely covered the surface at a coverage of about $2 \mathrm{cp}$ ML. 3D alloy clusters could be identified by the disruption that they cause to the substrate morphology following high temperature desorption of Co. Features remain at the alloy cluster sites on W(111) which resemble the alloy-induced mesas that have been observed on the W(110) surface. ${ }^{39}$

We assert that the changes of the (00) and (5/6 0) beam intensities at the characteristic times during deposition signal the completion of integral numbers of layers. The ratio of the characteristic times revealed in the evolution of the $(00)$ beam intensity, $t_{1}: t_{2}: t_{3}=1: 2: 3$, strongly suggests layered growth, although with some ambiguity about the number of layers deposited in the elemental time $t_{1}$. Coverage assignments are therefore made based upon the characteristic deposition times revealed by the $(00)$ and $(5 / 60)$ beams in the context of the atom densities of ps and cp Co films. The atom density of a $1 \mathrm{PML}=3 \mathrm{ML}$ ps film $\quad(17.30$ $\times 10^{14}$ atoms $/ \mathrm{cm}^{2}$ ), a single hcp Co (0001) bulk plane $\left(18.37 \times 10^{14}\right.$ atoms $\left./ \mathrm{cm}^{2}\right)$, and a $(6 \times 6)$ cp layer $(19.38$ $\times 10^{14}$ atoms $/ \mathrm{cm}^{2}$ ) are very similar. In particular, a single $(6 \times 6)$ cp layer contains $12 \%$ more material than a $3-\mathrm{ML}$ ps film. Therefore, nucleation of the $(6 \times 6)$ phase at $1.15 t_{1}$ is interpreted to mean that $t_{1}$ corresponds to deposition of $3 \mathrm{ps}$ ML. Given this assignment, the peak of the $(6 \times 6)$ diffraction at $t_{2}^{*}=1.08 t_{2}=2.16 t_{1}$ for deposition temperatures $T$ $\geqslant 710 \mathrm{~K}$ occurs at a coverage that is equivalent to $6.48 \mathrm{ps}$ ML. This is reasonably close to the content of two $(6 \times 6)$ layers, which is equivalent to $6.72 \mathrm{ps} \mathrm{ML}$, or to the content of one $(6 \times 6)$ layer and 3 ps ML, which is equivalent to 6.36 ps ML. LEEM observations confirm that the $(6 \times 6)$ phase nucleates and completely covers the surface (Fig. 4) within the same time frame that the $(6 \times 6)$ diffraction appears and reaches its peak (Fig. 2). Thus, the LEED $I(t)$ data indicate either that material in excess of $3 \mathrm{ps}$ ML transforms to a $(6 \times 6)$ layer on top of a $3-\mathrm{ML}$ ps buffer layer, or that all material present transforms so that two $(6 \times 6)$ cp layers are present at $t_{2}^{*}$. Due to experimental uncertainties, we cannot distinguish which of these two descriptions is correct on the basis of the characteristic deposition times. In principle, LEED $I(V)$ analysis may shed some light on the $(6 \times 6) \mathrm{cp}$ structure and the number of $\mathrm{cp}$ layers present at $t_{2}^{*}$. However, one drawback of doing LEED $I(V)$ measurements in a LEEM is that the range of momentum transfer parallel to the surface is limited to not much more than what is shown in Fig. 1. This limits the size of the data set, which consequently would increase the uncertainty of the results.

The assignment of $3 \mathrm{ps}$ ML to $t_{1}$ is also supported by diffraction intensity oscillations in SPLEEM experiments. Occasionally, weak oscillations of the integrated image intensity were observed at an imaging energy of $4.5 \mathrm{eV}$ during ps growth at $380 \mathrm{~K}$. These oscillations are caused by periodic nucleation, growth and coalescence during layer-by-layer growth. For the experiment depicted in Fig. 5, a deposition rate of $0.23 \mathrm{ps} \mathrm{ML/min}$ is determined from the oscillation period for 1 ps ML. Based upon this deposition rate, the breakpoint at $t_{3}^{\prime} \approx 39$ min in Fig. 5 occurs at a coverage of close to 9 ps ML. The break at $t_{3}^{\prime}$ in Fig. 5 also corresponds to the break that is indicated at $t_{3}$ in Fig. 2 for growth at 380 $\mathrm{K}$ at a higher deposition rate. Association of $t_{3}$ and $t_{3}^{\prime}$ with 9 ps ML coverage in Figs. 2 and 5 confirms that the times indicated as $t_{1}$ and $t_{2}$ in Fig. 2 correspond to 3 and 6 ps ML, respectively. The times $t_{1}$ and $t_{2}$ in Fig. 2 occur outside the time frame and coverage range of Fig. 5. The three distinct 
segments in the (00) beam $I(t)$ curves that occur between the start of deposition and $t_{1}$ at $T>685 \mathrm{~K}$ in Fig. 2 also suggest layer-by-layer growth of individual ps layers.

Our observations of the (00) beam intensity and only fleeting presence of $(5 / 60)$ intensity at $380 \mathrm{~K}$ indicate that thick ps films can be grown at this temperature. Given the coverage assignment stated above, our results demonstrate that ps films at least $15 \mathrm{ML}$ (5 PML) thick can be grown at $380 \mathrm{~K}$. This is consistent with the growth of thick ps Co films at room temperature achieved in earlier work. ${ }^{16}$ The pseudo layer-by-layer growth of such thick ps films may seem surprising in view of the large difference between the atomic diameters of $\mathrm{Co}$ and $\mathrm{W} \quad\left(d_{\mathrm{W}}=0.274 \mathrm{~nm}, d_{\mathrm{Co}}\right.$ $=0.251 \mathrm{~nm}$ ) and the first principles results in Table I. From Table I (and II), we see that one monolayer of Co wets the $\mathrm{W}(111)$ [and $\mathrm{W}(110)$ ] surfaces. If more than one pseudomorphic monolayer of Co is added to the $\mathrm{W}$ substrate, then the heat of formation becomes positive. This means that it is more favorable for the Co layer to form 3D macroscopic islands than to form a second 2D layer. Within the generalized gradient approximation (GGA) formalism, the equilibrium growth mode should thus be Stranski-Krastanov. There would be one wetting monolayer, followed by $3 \mathrm{D}$ island growth.

Upon closer inspection, pseudo-layer-by-layer growth of thick ps films at $380 \mathrm{~K}$ is understandable. When comparing different crystal structures of the same metal, the principle of constant atomic volume is usually well fulfilled. In hcp Co, the atomic volume is $0.0111 \mathrm{~nm}^{3}$. When the basal plane is expanded from 0.2507 to 0.4475 -nm interatomic distance to fit the $\mathrm{W}(111)$ periodicity, then the interplanar (0001) spacing has to be reduced by a factor of $(0.2507 / 0.4475)^{2}=0.3139$ from 0.204 to $0.064 \mathrm{~nm}$ in order to maintain the same atomic volume. This rough estimate of the ps Co interlayer spacing is supported by first principles calculations results in Table I. Keeping in mind that 3 ps Co monolayers contain nearly as many atoms as one hcp Co monolayer does $\left(17.30 \times 10^{14}\right.$ vs $18.37 \times 10^{14}$ atoms $/ \mathrm{cm}^{2}$, respectively), one may consider 3 ps Co monolayers on W(111) as one strongly corrugated and slightly (3.4\% laterally) expanded $\mathrm{Co}(0001)$ monolayer. With increasing thickness, the corrugation and lateral expansion are expected to decrease similar to the distortions in the $\mathrm{Co}(0001)$ layer on W(110), making three "pseudomorphic" Co layers on W(111) similar to the $\mathrm{Co}(0001)$ layer and energetically sufficiently favorable for kinetically-limited, metastable growth at low temperatures. Furthermore, the GGA calculation assumes a pseudomorphic overlayer, whereas slightly broadened $(1 \times 1)$ diffraction spots, indicative of some form of disorder, and a small $(6 \times 6) \mathrm{cp}$ component beyond 3 ps ML are found experimentally at $380 \mathrm{~K}$. These deviations from perfect ps registry may act as mechanisms for strain relief which promote metastable layer growth of the mostly ps film.

At higher deposition temperatures, kinetic growth limitations will be diminished and the onset of 3D cluster nucleation and growth will occur on thinner wetting layers. Eventually, the thermodynamically defined morphology (1 ps ML plus 3D clusters) should be expected if sufficiently high deposition temperature is used. Experimentally, we find that the recovery of the (00) beam intensity after $t_{2}(6 \mathrm{ps}$ ML) becomes dramatically less pronounced at deposition temperatures above $380 \mathrm{~K}$ until no recovery is seen at $685 \mathrm{~K}$ and above. We interpret this to be evidence of the formation of 3D clusters on top of a (meta-)stable wetting layer, whose thickness decreases dramatically in this temperature range. 3D clusters were not actually observed with LEEM, possibly because of small cluster size and also, at $685 \mathrm{~K}$, partly due to the low intensity of the (00) beam used for imaging after $t_{2}$. However, this interpretation is consistent with the observation by Guan et al. that thick, room temperature-deposited ps Co films agglomerate into 3D clusters on top of a wetting layer when annealed; this morphological change was most pronounced during annealing between 500 and $700 \mathrm{~K} .{ }^{16}$ This is precisely the temperature range that the prevalence of $3 \mathrm{D}$ clustering over layered growth of thick ps films is revealed by our LEED measurements. Our experimental results indicate that 6-ML-thick ps Co films are stable against clustering at $685 \mathrm{~K}$. This contradicts the earlier assumption of Guan et al. that ps films only half as thick were stable at this temperature and above. ${ }^{16}$ It is worth noting that Kolaczkiewicz and Bauer found for Ni/W(111) and Fe/W(111) that 3D clusters formed on top of stable four layer films at room temperature. ${ }^{14}$ An important difference between $\mathrm{Co}, \mathrm{Ni}$, and $\mathrm{Fe}$ is that the stable Co films are ps, whereas the $\mathrm{Ni}$ and $\mathrm{Fe}$ are ps for the first one and two layers, respectively, and then fcc(111) thereafter.

As the tendency for clustering on top of the pseudomorphic film increases between 380 and $685 \mathrm{~K}$, the $(6 \times 6)$ ordered component of the film also increases but remains relatively small in this temperature range. In the absence of the $\mathrm{cp}(6 \times 6)$ component, even fewer than 6 ps ML may be metastable at $685 \mathrm{~K}$. Development of the theoretically predicted, thermodynamically stable morphology at even higher deposition temperature is pre-empted by the more complete formation of the $\mathrm{cp}(6 \times 6)$ structure between 710 and $760 \mathrm{~K}$. The emergence of the cp structure is signalled by distinctly stronger $(6 \times 6)$ diffraction and a subtle change in the time, $t_{2}^{*}$, that the $(6 \times 6)$ diffraction spots reach their maximum intensity (Fig. 3). The changes in the $(6 \times 6)$ diffraction are also accompanied by a subtle change of the (00) beam $I(t)$ curve between $t_{1}$ and $t_{2}$ (Fig. 2) and by a change in the (00) intensity at $t_{1}$ in this temperature range [Fig. 3(a)]. Formation of the cp structure reflects the tendency for the overlayer to seek a compromise between the Co intralayer energy (2D layers always prefer to contract from the bulk lattice constants) and the interaction energy between Co and W (which usually favors registry with the substrate) instead of going to $3 \mathrm{D}$ islands. The drop of the $(5 / 60)$ intensity after its maximum at $t_{2}^{*}$, its broad minimum at $t_{4}$, and a subsequent slow increase (Fig. 2) indicate that the $(6 \times 6)$ film at least partially suppresses three-dimensional clustering at and above $685 \mathrm{~K}$. Because of the irreproducibility of the time $t_{4}$ and the fact that this time occurs later than would be expected for the completion of an additional cp layer, we believe that some $3 \mathrm{D}$ clustering still occurs on top of the $(6 \times 6) \mathrm{cp}$ layer between 685 and $810 \mathrm{~K}$. At $T \geqslant 840 \mathrm{~K}$, however, the $(5 / 60)$ diffraction intensity appears to decrease more slowly after reaching a peak at $t_{2}^{*}$. This indicates that $3 \mathrm{D}$ clustering on 
top of a stable cp layer is more significant than at lower temperatures. It is worth noting that clustering is initiated at nearly the same deposition time on the ps layers at intermediate temperature and $(6 \times 6) \mathrm{cp}$ layers at high temperature. This means that roughly the same amount of Co is present in the ps and cp 2D wetting layers.

\section{B. Magnetism}

The potential impact of substrate surface steps on thin film magnetism is important in the context of magnetic filmcoated tips because steps are a significant part of tip morphology. The equilibrium shape of a tip in first approximation is a hemisphere on a conical shank. This will be modified as the result of tip sharpening procedures. Field ion and field emission microscopy show that a small triangular (111) plane bounded by three facet planes is present at the apex of sharpened (111)-oriented tips. ${ }^{40,41}$ The (111) plane is cut at its edges by three $\{112\}$ planes in the theoretical $\mathrm{W}$ equilibrium crystal shape. ${ }^{8}$ If there is a curved region on a tip between the (111) plane and adjacent (112) facet, then it will comprise steps that run along the [1 $\overline{1} 0]$ direction. Therefore, the magnetic measurements performed here on well-oriented $\mathrm{W}(111)$ and vicinal W(111) with step edges nearly along the surface $[1 \overline{1} 0]$ direction may give important clues about the impact of tip morphology upon magnetism in Co-coated $\mathrm{W}(111)$ tips.

An important aspect of ultrathin film magnetism is the magnetization direction, which is influenced by a number of factors including magnetocrystalline anisotropy, shape anisotropy, and epitaxial strain. A knowledge of the magnetization direction is crucial for predicting the spin polarization direction of electrons emitted by tunneling from ultrathin film-coated tips. Although only spontaneous in-plane magnetization was observed on the well-oriented and vicinal surfaces, it cannot be ruled out entirely that Co/W(111) also has an out-of-plane magnetization component similar to Co films on $\mathrm{W}(110){ }^{42}$ The SPLEEM studies were made with a large field of view because of the large domain size, so that small out-of-plane magnetization features may have been lost in the noise. Magnetization in the in-plane $\langle 11 \overline{2}\rangle$ and $\langle 1 \overline{1} 0\rangle$ directions cannot be understood on the basis of what is known about magnetocrystalline anisotropy in bcc Co. The [001] direction is easy axis, the [111] is intermediate, and the [110] is hard axis in bcc Co stabilized on $\operatorname{GaAs}(110) .{ }^{43}$ It was pointed out that the magnetic anisotropy in bcc $\mathrm{Co} / \mathrm{GaAs}$ cannot be so easily understood in terms of the sign of the anisotropy constant $K_{1} \cdot{ }^{44}$ In particular, [001] is easy, [110] is intermediate and [111] is hard for bcc $\alpha$-Fe, whereas [111] is easy, [110] is intermediate and [001] is hard for fcc $\mathrm{Ni}^{45}$ The difference between $\alpha-\mathrm{Fe}$ and $\mathrm{Ni}$ is related to the sign of $K_{1}$, which is positive for bcc $\alpha$-Fe and negative for fcc Ni.

Given that the out-of-plane layer spacing in ps Co/W(111) is strongly contracted, description of this system as bcc is not well motivated and comparison to hcp Co may be more appropriate. For hexagonal structure, the magnetocrystalline anisotropy energy is expressed as ${ }^{46}$
$E=K_{0}+K_{1} \sin ^{2} \theta+K_{2} \sin ^{4} \theta+K_{3} \sin ^{6} \theta+K_{4} \sin ^{6} \theta \cos 6 \varphi$,

where $\theta$ is the angle between the magnetization and the $c$ axis, and $\phi$ is the angle in the basal plane relative to one of

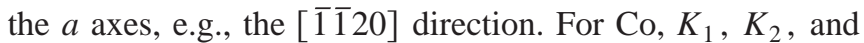
$K_{3}$ are positive at room temperature which determines the easy $c$ axis. ${ }^{45,46,47}$ The in-plane anisotropy constant $K_{4}$ has been determined to be positive and about 100 times smaller than the dominant $K_{1}$ at room temperature. ${ }^{47}$ In the absence of the $c$-axis anisotropy, the easy magnetization directions would be the three equivalent hexagonal in-plane [10 $\overline{1} 0]$, [1100], and [0110] directions, which are at $30^{\circ}$ from the $a$ axes. ${ }^{47}$ If we treat 3 ps ML Co/W(111) as a corrugated $\mathrm{Co}(0001)$ plane, then the in-plane easy hcp axes correlate

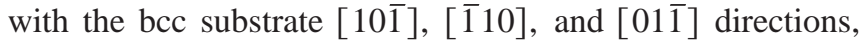
which happen also to be the directions between nearest neighbors in the $\mathrm{W}(111)$ plane. While this can explain the observation of magnetization along the in-plane $\langle 1 \overline{1} 0\rangle$ directions on the well-oriented $\mathrm{W}(111)$ surface, magnetization along the in-plane $\langle 11 \overline{2}\rangle$ directions which are equivalent to the hexagonal $a$-axis directions in the overlayer is not accounted for by the hexagonal anisotropy energy. The presence of uniaxial magnetization along one of the three inplane $\langle 11 \overline{2}\rangle$ directions that is nearly perpendicular to step edges in Co on the vicinal W(111) surface suggests that steps cause the magnetization to lie along this direction. On a welloriented surface, flat terraces are expected. But, it is nevertheless unavoidable to have a hill-and-valley morphology with local regions having a step density that is higher than expected from the small sample misorientation from the (111) plane. Therefore, the presence of magnetization along the $\langle 11 \overline{2}\rangle$ in-plane directions on the well-oriented surface is also believed to be step induced. The sensitivity of the basal plane anisotropy to external influences is not unprecedented. A uniaxial basal plane anisotropy in bulk hcp Co, which was found instead of the expected three-fold one, was explained by lattice deformation induced anisotropy. ${ }^{48}$ In light of the small basal plane magnetocrystalline anisotropy constant $K_{4}$ in bulk $\mathrm{Co}$, we expect that the magnetocrystalline anisotropy in ps $\mathrm{Co} / \mathrm{W}(111)$ is small as well. Absence of perpendicular magnetization in $\mathrm{Co} / \mathrm{W}(111)$ is due to the shape anisotropy.

Another fundamental issue in the magnetism of ultrathin films is how magnetic ordering is affected by their reduced dimension. A finite size scaling law has been found to describe the suppression of the Curie temperature in several thin films systems. ${ }^{13,31,49,50}$ From the standpoint of fabricating magnetic film-coated $\mathrm{W}$ tips for vacuum tunneling applications, it is important to know the thickness that films are magnetic at room temperature. For ps Co films on W(111), the onset of magnetism occurs at $7.60 \pm 0.15 \mathrm{ps} \mathrm{ML}$ at $380 \mathrm{~K}$, which is equivalent to $2.39 \mathrm{Co}(0001)$ layers, and was not fundamentally altered by surface steps. This is somewhat larger than the onset of magnetism in $1.7 \mathrm{Co}(0001) \mathrm{cp} \mathrm{ML}$ on $\mathrm{W}(110)$ at room temperature. ${ }^{51,52}$ The Curie temperature for $\mathrm{Co} / \mathrm{W}(110)$ exceeded $380 \mathrm{~K}$ for thickness above $1.9 \mathrm{cp}$ ML. ${ }^{52}$

For Co/W(110), an extrapolation to zero magnetic moment at 0.9 (0001) cp ML at room temperature ${ }^{53}$ and an 
extrapolation of the Curie temperature to $0 \mathrm{~K}$ at 1.1 (0001) cp ML have been reported. ${ }^{52}$ If these extrapolations are valid, then they would indicate that the magnetic moments on the Co atoms in immediate contact with $\mathrm{W}$ are quenched. This is plausible because the electronic structure of the first monolayer differs strongly from that of bulk Co. Due to hybridization between the Co $3 d$ and W $5 d$ bands, the Co $3 d$ bands are shifted to lower energies so that the minority spin bands become strongly occupied. ${ }^{54}$ This reduces the magnetic moment, possibly to zero, a phenomenon that is expected to occur on most interfaces at which strong interactions between the ferromagnetic film and the substrate cause a sufficient shift of the band structure to lower energies.

From our calculation results in Tables I and II, we see that the binding of 1 and 2 ps ML are stronger on W(111) than $\mathrm{W}(110)$. This is understandable since (111) is a more open surface and the $\mathrm{W}$ surface atoms are more reactive than those on the more close-packed (110) orientation. The distance between the Co atoms and the $\mathrm{W}$ surface layer is also shorter in the (111) orientation. As a result, there is stronger interaction and hybridization between the Co and $\mathrm{W}$ atomic orbitals on the W(111) surface, leading to the stronger binding. Nevertheless, it is interesting to note that the first Co layer that is in direct contact with $\mathrm{W}$ remains magnetic at $T=0$, according to theory, despite the strong hybridization between Co and the nonmagnetic $\mathrm{W}$ substrate. The magnetic moment of this Co layer is diminished with respect to the bulk value, which is calculated to be $1.70 \mu_{B}$. The magnetic moment of the first Co layer is smaller on (111) than (110), consistent with the stronger interaction and smaller distance between the Co and W substrate in the (111) orientation. Therefore, on the basis of these calculations, monolayer Co on W(110) and W(111) are expected to be ferromagnetically ordered at $T=0$. The onset of magnetism near room temperature in ps $\mathrm{Co} / \mathrm{W}(111)$ at slightly larger (equivalent-cp) thickness than in cp Co layers on $\mathrm{W}(110)$ may be related to the reduced magnetic moments on Co at the W(111) interface, or to the coupling constants between moments, which are not addressed in our calculations.

\section{SUMMARY AND CONCLUSIONS}

Summarizing the key results, we find that Co grows on the $\mathrm{W}(111)$ surface in either ps or $(6 \times 6) \mathrm{cp}$ structure depending upon deposition temperature. The cp structure has not been identified previously. Given that the out-of-plane layer spacing in ps $\mathrm{Co} / \mathrm{W}(111)$ is expected to be strongly contracted, 3 ps ML may be considered to be a highly corrugated and slightly laterally expanded $\mathrm{Co}(0001)$ layer. Calculated relaxation geometries for the first 3 ps ML support this picture. Thus, thick, metastable ps films can be grown near room temperature, in agreement with the earlier work of Guan et al. ${ }^{16}$ The growth mode changes from pseudo layerby-layer to Stranski-Krastanov above $380 \mathrm{~K}$ and the tendency to form a $(6 \times 6) \mathrm{cp}$ structure beyond 3 ps ML increases but remains small up to $685 \mathrm{~K}$. Although experiment indicates that the equivalent of $6 \mathrm{ps}$ ML wets the surface at $685 \mathrm{~K}$, our first principles calculations indicate that only $1 \mathrm{ps}$ ML is thermodynamically stable against agglomeration into three-dimensional clusters. Both of these results contradict the assumption made earlier that the stable wetting layer consists of 3 ps Co ML on W(111). ${ }^{16}$ A 6-ML film may be stabilized at $685 \mathrm{~K}$ by the presence of the cp component. Agglomeration of material in excess of 1 ps ML is preempted above $710 \mathrm{~K}$ by the more complete formation of the $\mathrm{cp}$ structure. Although complete formation of the cp structure at least partially suppresses agglomeration, StranskiKrastanov growth again prevails above $840 \mathrm{~K}$; two stable cp ML or one cp ML atop 3 ps ML are indicated. Threedimensional alloying is found at significantly higher Co coverage at these temperatures.

Pseudomorphic films with thickness above 7.6 ML, equivalent to $2.4 \mathrm{Co}(0001) \mathrm{cp}$ layers, are ferromagnetic at $380 \mathrm{~K}$ with an in-plane magnetization. Calculations indicate that Co moments are significantly smaller on W(111) than on $\mathrm{W}(110)$, as would be expected from stronger hybridization on the atomically rougher $\mathrm{W}(111)$ surface. This result is also consistent with the experimental observation that the onset of ferromagnetism near room temperature occurs in slightly thicker films on W(111) than on W(110). Spontaneous magnetization in the in-plane $\langle 1 \overline{1} 0\rangle$ directions in Co on a welloriented $\mathrm{W}(111)$ surface is accounted for by the basal plane magnetocrystalline anisotropy of bulk hcp Co. Magnetization in the in-plane $\langle 11 \overline{2}\rangle$ directions on the well-oriented and vicinal $\mathrm{W}(111)$ surfaces is attributed to a step-induced anisotropy. The strong influence of substrate atomic steps that is seen in the magnetization easy axis and magnetic domain structure suggests that the in-plane magnetocrystalline anisotropy is small, analogous to the basal plane of bulk Co.

Observations pertaining to the structure, morphology, and magnetism of $\mathrm{Co} / \mathrm{W}(111)$ taken together lead us to conclude that Co-coated W(111) tips would be viable for obtaining spin polarization in vacuum tunneling in field emission and STM. By carrying out Co deposition near room temperature, Stranski-Krastanov growth can be avoided and continuous films are obtained that are of sufficient thickness to be magnetic at room temperature. Furthermore, these growth conditions avoid alloying between $\mathrm{Co}$ and $\mathrm{W}$ that would be expected to be detrimental to their magnetic properties. A transverse spin polarization in vacuum tunnelling is expected, but the strong influence of steps on the film magnetization directions leads us to expect that tip morphology will have a strong impact upon the azimuthal orientation of the emitted spin. This could be a disadvantage for reproducibility or an advantage for spin manipulation. Further work is encouraged to test these ideas.

\section{ACKNOWLEDGMENTS}

M.S.A. is grateful to Ming L. Yu for valuable discussions. This work was supported by the Hong Kong Research Grants Council under Grant Nos. HKUST6137/00P and HKUST6152/01P (K.L.M., C.T.C., and M.S.A), by the National Science Foundation under Grant No. DMR9818296 (R.Z. and E.B.), the National Science Council Grant No. 90-2112-M-194-019 and a grant of computer time at the National Center for High Performance Computing (T.C.L and S.F.H). 
${ }^{1}$ M. Landoldt and M. Campagna, Phys. Rev. Lett. 38, 663 (1977).

${ }^{2}$ M. Landoldt and Y. Yafet, Phys. Rev. Lett. 40, 1401 (1978).

${ }^{3}$ G. Chrobok, M. Hoffmann, G. Regenfus, and R. Sizmann, Phys. Rev. B 15, 429 (1977).

${ }^{4}$ M. Pratzer, H. J. Elmers, M. Bode, O. Pietzsch, A. Kubetzka, and R. Wiesendanger, Phys. Rev. Lett. 87, 127201 (2001).

${ }^{5}$ O. Pietzsch, A. Kubetzka, M. Bode, and R. Wiesendanger, Phys. Rev. Lett. 84, 5212 (2000).

${ }^{6}$ G. L. Kellogg, in Physics of Solid Surfaces, edited by G. Chiarotti, Landolt-Börnstein, New Series, Group III, Vol. 24, pt. d (Springer, Berlin, 1996), p. 352.

${ }^{7}$ T. M. Gardiner, H. M. Kramer, and E. Bauer, Surf. Sci. 112, 181 (1981).

${ }^{8}$ J. G. Che, C. T. Chan, C. H. Kuo, and T. C. Leung, Phys. Rev. B 57, 1875 (1998).

${ }^{9}$ M. Drechsler, in Surface Mobilities on Solid Materials, edited by V. T. Binh (Plenum, New York, 1981), p. 405.

${ }^{10}$ For an early review, see E. Bauer, in The Chemical Physics of Solid Surfaces and Heterogeneous Catalysis, edited by D. A. King and D. P. Woodruff (Elsevier, Amsterdam, 1984), Vol. 3, pt. B.

${ }^{11}$ E. Bauer, J. Phys.: Condens. Matter 11, 9365 (1999), and references therein.

${ }^{12}$ T. Duden, R. Zdyb, M. S. Altman, and E. Bauer, Surf. Sci. 480, 145 (2001).

${ }^{13}$ H.-J. Elmers, Int. J. Mod. Phys. B 9, 3115 (1995).

${ }^{14}$ J. Kolaczkiewicz and E. Bauer, Surf. Sci. 420, 157 (1999).

${ }^{15}$ C. Dong, L. Zhang, U. Diebold, and T. E. Madey, Surf. Sci. 322, 221 (1995).

${ }^{16}$ J. Guan, R. A. Campbell, and T. E. Madey, Surf. Sci. 341, 311 (1995).

${ }^{17}$ H. S. Tao, J. E. Rowe, and T. E. Madey, Surf. Sci. 407, L640 (1998).

${ }^{18}$ T. E. Madey, J. Gaun, C. H. Nien, C. Z. Dong, H. S. Tao, and R. A. Campbell, Surf. Rev. Lett. 3, 1315 (1996).

${ }^{19}$ T. E. Madey, J. Guan, C. Z. Dong, and S. M. Shivaprasad, Surf. Sci. 287/288, 826 (1993).

${ }^{20}$ S. A. Shakirova and E. V. Serova, Surf. Sci. 422, 24 (1999).

${ }^{21}$ F. M. Gonchar, T. P. Smereka, S. I. Stepanovskii, and G. V. Babkin, Fiz. Tverd. Tela (Leningrad) 30, 3541 (1988) [Sov. Phys. Solid State 30, 2035 (1988)].

${ }^{22}$ G. M. Bliznakov, T. S. Marinova, and A. D. Popov, Izv. Khimiya 9, 203 (1976).

${ }^{23}$ C. H. Nien, T. E. Madey, Y. W. Tai, T. C. Leung, J. G. Che, and C. T. Chan, Phys. Rev. B 59, 10355 (1999).

${ }^{24}$ J. G. Che, C. T. Chan, C. H. Kuo, and T. C. Leung, Phys. Rev. Lett. 79, 4230 (1997).

${ }^{25}$ J. G. Che and C. T. Chan, Surf. Sci. 401, L432 (1998).

${ }^{26}$ E. Bauer, Rep. Prog. Phys. 57, 895 (1994).

${ }^{27}$ T. Duden and E. Bauer, J. Electron Microsc. 47, 379 (1998).

${ }^{28}$ W. F. Chung and M. S. Altman, Ultramicroscopy 74, 237 (1998).

${ }^{29}$ M. S. Altman, H. Pinkvos, J. Hurst, G. Marx, H. Poppa, and E. Bauer, in Magnetic Materials: Microstructure and Properties, edited by T. Suzuki, MRS Symposia Proceedings, No. 232 (Materials Research Society, Pittsburgh, 1991) p. 125.

${ }^{30}$ T. Duden and E. Bauer, Rev. Sci. Instrum. 66, 2861 (1995).

${ }^{31}$ K. L. Man, M. S. Altman, and H. Poppa, Surf. Sci. 480, 163 (2001).

${ }^{32}$ T. Scheunemann, R. Feder, J. Henk, E. Bauer, T. Duden, H. Pinkvos, H. Poppa, and K. Wurm, Solid State Commun. 104, 787 (1997).

${ }^{33}$ P. Blaha, K. Schwarz, and J. Luitz, WIEN97 (Vienna University of Technology, 1997). Improved and updated Unix version of original copyrighted wIEN code by P. Blaha, K. Schwarz, P. Soratin, and S. B. Trickey, Comput. Phys. Commun. 59, 399 (1990).

${ }^{34}$ J. P. Perdew, J. A. Chevary, S. H. Vosko, K. A. Jackson, M. R. Pederson, D. J. Singh, and C. Fiolhais, Phys. Rev. B 46, 661 (1992); J. P. Perdew, K. Burke, and M. Ernzerhof, Phys. Rev. Lett. 77, 3865 (1996).

${ }^{35}$ R. M. Bozorth, Ferromagnetism (IEEE Press, Piscataway, NJ, 1993), p. 264.

${ }^{36}$ N. A. W. Holzwarth, J. A. Chervenak, C. J. Kimmer, Y. Zeng, W. Xu, and J. Adams, Phys. Rev. B 48, 12136 (1993).

${ }^{37}$ C. J. Fall, N. Binggeli, and A. Baldereschi, Phys. Rev. B 65, 045401 (2001).

${ }^{38}$ T. E. Madey (private communication).

${ }^{39}$ H. Pinkvos, H. Poppa, E. Bauer, and G. M. Kim, in Magnetism and Structure of Systems in Reduced Dimension, edited by R. F. C. Farrow, B. Dieny, M. Donath, A. Fert, and B. D. Hermsmeier (Plenum, New York, 1993), pp. 25-31.

${ }^{40}$ H. S. Kim, M. L. Yu, U. Staufer, L. P. Muray, D. P. Kern, and T. H. P. Chang, J. Vac. Sci. Technol. B 11, 2327 (1993).

${ }^{41}$ M. L. Yu, B. W. Hussey, H. S. Kim, and T. H. P. Chang, J. Vac. Sci. Technol. B 12, 3431 (1994).

${ }^{42}$ T. Duden and E. Bauer, Phys. Rev. Lett. 77, 2308 (1996).

${ }^{43}$ G. A. Prinz, Phys. Rev. Lett. 54, 1051 (1985).

${ }^{44}$ Y. Z. Wu, H. F. Ding, C. Jing, D. Wu, G. L. Liu, V. Gordon, G. S. Dong, and X. F. Jin, Phys. Rev. B 57, 11935 (1998).

${ }^{45}$ E. P. Wohlfarth, in Ferromagnetic Materials, edited by E. P. Wohlfarth (Elsevier, Amsterdam, 1980), Vol. 1.

${ }^{46}$ M.B. Stearns, in Magnetic Properties of Metals, edited by H.P.J. Wijn, Landolt-Börnstein, New Series, Group III, Vol. 19, pt. a (Springer, Berlin, 1986), p. 41.

${ }^{47}$ D. M. Paige, B. Szpunar, and B. K. Tanner, J. Magn. Magn. Mater. 44, 239 (1984).

${ }^{48}$ N. P. Barradas, M. Rots, A. A. Melo, and J. C. Soares, Phys. Rev. B 47, 8763 (1993).

${ }^{49}$ M. Farle, K. Baberschke, U. Stetter, A. Aspelmeier, and F. Gerhardter, Phys. Rev. B 47, 11571 (1993).

${ }^{50} \mathrm{U}$. Gradmann, in Handbook of Magnetic Materials, edited by K. H. J. Buschow (Elsevier, Amsterdam, 1993), Vol. 7.

${ }^{51}$ J. Kohlhepp, Ph.D. thesis, TU Clausthal, 1994.

${ }^{52}$ G. Garreau, M. Farle, E. Beaurepaire, and K. Baberschke, Phys. Rev. B 55, 330 (1997).

${ }^{53}$ H. Fritzsche, J. Kohlhepp, and U. Gradmann, Phys. Rev. B 51, 15933 (1995).

${ }^{54}$ H. Knoppe and E. Bauer, Phys. Rev. B 48, 1794 (1993). 